\title{
Re-Excision Rate in Breast Conservation Surgery after Neoadjuvant Chemotherapy
}

\author{
Jung Hyun Song, Jeong Yeong Park, Jung Eun Choi, Suhwan Kang, Soo Jung Lee, Youngkyung Bae ${ }^{1}$ \\ Departments of Surgery and ${ }^{1}$ Pathology, Yeungnam University Hospital, Daegu, Korea
}

Purpose: The purpose of this study was to compare the success rate of re-excision and breast-conserving surgery (BCS) between patients who received neoadjuvant chemotherapy and those who did not. Methods: In this retrospective cohort study, 256 women who had clinical T2 breast cancer and planned to receive, as initial treatment either BCS $(n=197)$ or neoadjuvant chemotherapy $(n=59)$ between January 2009 and December 2012 were included. The data, including age, initial tumor size, mammographic microcalcification, ultrasound multifocality and axillary nodal status, were collected. The pathologic tumor size, p-multifocality, histologic type, estrogen receptor, progesterone receptor, human epidermal growth factor receptor 2, Ki-67, ductal carcinoma in situ (DCIS) and extensive intraductal component (EIC) were also reviewed. The re-excision and BCS success rates were investigated. Univariate analysis and regression model were used. To reduce the effect of selection bias, propensity score matching-based analysis was also performed. Results: Of the 256 patients, 178 patients $(90.4 \%$, $178 / 197)$ in the non-neoadjuvant group and 56 patients $(94.9 \%, 56 / 59)$ in the neoadjuvant group received BCS $(p=0.406)$. In propensity-matched cohorts $(n=118)$, the re-excision rate was similar in the two groups $(35.6 \%$ in neoadjuvant group vs. $35.6 \%$ in non-neoadjuvant group, $p=1.000)$. BCS success rate was slightly higher in neoadjuvant group (94.9\%, 56/59) than in non-neoadjuvant group (86.4\% [51/59], $p=0.205$ ). In logistic regression model, clinicopathologic factors associated with re-excision were pathologic multifocality (odds ratio [OR], 4.56; $p=0.0142$ ), high Ki-67 ( $\geq 50 \%$ ) (OR, $0.7 ; p=0.0243$ ) and DCIS component (OR, 2.67; $p=0.0261)$. Conclusion: This study showed that neoadjuvant chemotherapy could increase the success rate of BCS but could not decrease that of re-excision. The re-excision rate is more associated with pathologic finding rather than the effect of neoadjuvant chemotherapy.

Key Words: Breast neoplasms, Margins of excision, Neoadjuvant chemotherapy, Re-excision, Segmental mastectomy

\section{INTRODUCTION}

Many studies report no difference of survival outcome between breast-conserving surgery (BCS) followed by radiotherapy and mastectomy. BCS followed by radiotherapy is a standard treatment in breast cancer [1-4]. For a successful BCS, the primary objective is to achieve negative resection. Margin status is among the most important predictors of increased risk for loco-regional recurrence [5-7]. The secondary goal is to provide a satisfactory cosmetic outcome. However, as the resection rate is increased to obtain a negative resection margin, more tissues are removed. This leads to a decrease in cosmetic satisfaction and provides a factor to switch from BCS to mastectomy, thus re-resection is important in breast operation $[8,9]$.

Correspondence: Soo Jung Lee

Department of Surgery, Yeungnam University Hospital, 170 Hyeonchung-ro, Nam-gu, Daegu 42415, Korea

Tel: +82-53-620-3587, Fax: +82-53-624-1213, E-mail: crystallee@med.yu.ac.kr Received: Feb 17, 2017 Revised: Jun 8, 2017 Accepted: Jun 8, 2017
Recently, the use of neoadjuvant chemotherapy (NAC) in the treatment of breast cancer patients has increased. Preoperative chemotherapy reduces the size of the tumor, providing an opportunity to avoid mastectomy and increase the incidence of BCS. It can also be used to monitor the effectiveness of chemotherapy regimen in vivo. Because of these advantages, the use of preoperative chemotherapy is expected to increase, despite no difference in the survival and recurrence rates between preoperative chemotherapy and initial operation.

Unfortunately, the regression pattern of breast cancer after preoperative chemotherapy varies, it can occur in a mosaic pattern rather than circumferential pattern. This variation leads to re-excision, thus the success rate of BCS in primary chemotherapy (PC) varies [10-13]

Studies evaluating the success rate of BCS and re-excision rate after NAC are limited. The purpose of this study is to compare the re-excision rate and BCS success rate between patients who received NAC and those who did not. Another goal is to identify other variables that might be associated with margin status. 


\section{METHODS}

\section{Patient selection}

From January 2009 to December 2012, we analyzed the data of 1,892 patients treated for invasive breast cancer in Yeungnam University Hospital through a retrospective study with Institutional Review Board approval. Exclusion criteria were male breast cancer, ductal carcinoma in situ (DCIS), phyllodes tumor, lymphoma, sarcoma, secondary neoplasm, distant metastasis, tumor size $\leq 2 \mathrm{~cm}$ or $>5 \mathrm{~cm}$, skin edema, diffuse microcalcification and contraindications to radiotherapy. A total of 256 female patients who had clinical T2 invasive carcinoma and planned to receive, as initial treatment, either BCS or NAC were included. Of the 256, 59 patients received NAC and 197 patients were initially treated with BCS.

If the size of the cancer does not decrease despite the use of preoperative chemotherapy, then surgery was performed. If the tumor decreased and became difficult to palpate, under ultrasound or mammography, then a guided-wire was inserted by a radiologist on the day of operation. Before NAC, sentinel lymph node biopsy was performed. If axillary lymph node metastases were found in preoperative pathologic diagnoses, such as core needle biopsy and needle aspiration cytology or intraoperative frozen biopsy, complete axillary dissection was performed.

Preoperative chemotherapy regimens were docetaxel/carboplatin (TC), doxorubicin/cyclophosphamide (AC), cyclophosphamide/ methotrexate/fluorouracil (CMF), fluorouracil/epirubicin/cyclophosphamide (FEC), docetaxel+pharmorubicin and docetaxel/doxorubicin/cyclophosphamide (TAC). Patients who underwent preoperative hormonal therapy or target therapy were excluded.

This study meets the standards of ethical approval of the Yeungnam University Hospital (IRB file number: 2017-01-027).

\section{Data collection}

Clinical data, including age, preoperative or initial clinical tumor size, mammographic microcalcifications, ultrasound multifocality and axillary nodal status, were collected retrospectively. In the specimen obtained from BCS, the pathologic tumor size, multifocality, histologic type, hormone receptor and human epidermal growth factor receptor 2 (HER2)-neu status, Ki-67, DCIS and extensive intraductal component (EIC) were reviewed. The threshold for estrogen and progesterone receptor positivity was 10\%. HER2-neu status was assessed using the clonal immunohistochemical markers 4B5 and Tab 250. The patients evaluated as "doubtful” were analyzed via fluorescent in situ hybridization. High Ki-67 was defined as $>50 \%$ presentation in Ki-67.

\section{Margin and re-excision}

After lumpectomy in the operating room, the medial, superior, lateral and inferior resection margins were suture tagged and immediate margin status was confirmed via intraoperation frozen biopsy. Re-excision was performed when tumor cells were present in the resection margin or within $1 \mathrm{~mm}$. When the tumor cell was observed more than $2 \mathrm{~mm}$ apart or tumor-free resection margin was obtained, the operation was terminated. Mastectomy was performed when a tumor cell existed on the margin despite three or more re-excisions or when there residual volume is not enough to perform BCS. Permanent biopsy revealed a close margin of less than $1 \mathrm{~mm}$ or a tumor cell in the margin, and re-operation was performed to obtain a tumor-free resection.

\section{Statistical analysis}

The characteristics of the patients in both groups were analyzed via univariate analysis. The re-resection rate and success rate of BCS between the two groups were also examined. A selection bias of surgeons in the decision of treatment methods existed between the two groups because this is a retrospective study. To reduce the effect of selection bias, propensity score matching-based analysis was also performed. Clinicopathologic risk factors associated with re-excision were also examined using logistic regression models. The results were analyzed using SPSS version 21.0 for Windows (IBM Corp., Armonk, USA) and R 3.0.2 (http://www.web-r.org) with package "MatchIt" and "Survival." A $p$-value $<0.05$ was considered statistically significant.

\section{RESULTS}

Univariate analysis of patient characteristics: primary surgery (initial operation) versus primary chemotherapy (NAC)

In the univariate analysis, histologic type, hormone receptor status, HER2-neu status, and Ki-67 were similar between the two groups. 
Re-resection was lower in the primary surgery (PS) group (PS, 67/197 [34.0\%]; PC, 21/59 [35.6\%]; $p=0.946)$. Meanwhile, the BCS success rate was higher in the PC group (PS, 178/197 [90.4\%]; PC, 56/59 [94.9\%]; $p=0.406)$. However, statistical significance was not observed. Significant differences were noted in age $(p<0.001)$, clinical multifocality $(p=0.002)$, clinical tumor size $(p<0.001)$, pathologic tumor size $(p<0.001)$, DCIS $(p=0.001)$, and EIC $(p=0.049)$ (Table 1$)$.

In propensity score matching, univariate analysis of patients' characteristics

A selection bias of surgeons in the decision of treatment methods existed between the two groups because this is a retrospective study. A significant difference was noted in the age, clinical tumor size, and multifocality of the two groups before treatment. To reduce the effect of selection bias, propensity score matching-based analysis was also performed. Fifty-nine patients in primary surgery were enrolled as controls. A total of 118 patients were analyzed via univariate analysis (Table 2). Significant differences were noted in pathologic tumor size $(p<0.001)$, DCIS $(p=0.016)$, and EIC $(p=0.027)$ between the two groups. No difference was found between the two groups in terms of

Table 1. Univariate analysis of patients' characteristics

\begin{tabular}{lccr}
\hline Characteristic & PS $(n=197)$ & PC $(n=59)$ & \\
& No. $(\%)$ & No. $(\%)$ & \\
\hline Age $(y r)^{*}$ & $49.0 \pm 9.3$ & $43.6 \pm 7.5$ & $<0.001$ \\
C-microcalcification & $57(28.9)$ & $17(28.8)$ & 1.000 \\
C-multifocality & $44(22.3)$ & $26(44.1)$ & 0.002 \\
C-tumor size $(\mathrm{cm})^{\dagger}$ & $2.3[2.0,2.7]$ & $2.7[2.5,3.5]$ & $<0.001$ \\
Nodal metastasis & $82(41.6)$ & $29(49.2)$ & 0.382 \\
Pathologic size $(\mathrm{cm})^{\dagger}$ & $2.3[1.9,2.6]$ & $1.5[0.6,2.2]$ & $<0.001$ \\
Pathologic multifocality & $19(9.6)$ & $6(10.2)$ & 1.000 \\
Poor differentiation histology & $9(4.6)$ & $1(1.7)$ & 0.538 \\
ER (+) & $127(64.5)$ & $39(66.1)$ & 0.940 \\
PR (+) & $119(60.4)$ & $32(54.2)$ & 0.488 \\
HER2 (+) & $47(23.9)$ & $14(23.7)$ & 1.000 \\
Ki-67 $(\geq 50 \%)$ & $67(34.0)$ & $19(32.2)$ & 0.920 \\
DCIS (+) & $19(32.2)$ & $26(44.1)$ & 0.001 \\
EIC (+) & $41(20.8)$ & $5(8.5)$ & 0.049 \\
Re-excision & $67(34.0)$ & $21(35.6)$ & 0.946 \\
Success of BCS & $178(90.4)$ & $56(94.9)$ & 0.406 \\
\hline
\end{tabular}

$\mathrm{PS}=$ primary surgery; $\mathrm{PC}=$ primary chemotherapy; $\mathrm{C}$-microcalcification = clinical microcalcification; C-multifocality= clinical multifocality (ultrasound); C-tumor size = clinical tumor size (ultrasound); ER=estrogen receptor; $\mathrm{PR}=$ progesterone receptor; $\mathrm{HER} 2$ = human epidermal growth factor receptor 2-neu status; $\mathrm{DCIS}=$ ductal carcinoma in situ; $\mathrm{EIC}=$ extensive intraductal component; $\mathrm{BCS}=$ breast-conserving surgery.

${ }^{*}$ Mean $\pm \mathrm{SD}$; ${ }^{\dagger}$ Performs a normality test, forces analysis as non-normal. re-excision $(p=0.100)$. The success rate of BCS was slightly higher in primary chemotherapy but not statistically significant (PS, 52/59 [88.1\%] vs. PC, 56/59 [94.9\%]; $p=0.321)$.

\section{In propensity score matching, re-excision versus}

\section{non-re-excision}

In propensity score matching, 42 patients underwent re-excision out of the 118 patients (Table 3). In terms of re-excision, the success rate of BCS was slightly higher in primary chemotherapy $(94.9 \%$ [56/59]) than in primary surgery group (86.4\% [51/59]), but no statistical difference was noted $(p=0.205)$, whereas significant differences were noted in Ki-67 ( $p=0.014)$ and DCIS $(p=0.007)$. However treatment, primary chemotherapy versus primary surgery, between the two groups was not significantly different thus NAC appeared to have no effect on re-excision.

\section{In propensity score matching, logistic regression model}

Univariate and multivariate logistic regression models were used to examine the variables associated with re-excision. In the final logistic

Table 2. In propensity score matching, univariate analysis of patients' characteristics

\begin{tabular}{|c|c|c|c|}
\hline Characteristic & $\begin{array}{c}\text { PS }(n=59) \\
\text { No. }(\%)\end{array}$ & $\begin{array}{c}\mathrm{PC}(\mathrm{n}=59) \\
\text { No. }(\%)\end{array}$ & $p$-value \\
\hline Age $(y r)^{*}$ & $42.2 \pm 8.4$ & $43.6 \pm 7.5$ & 0.328 \\
\hline C-microcalcification & $20(33.9)$ & $17(28.8)$ & 0.691 \\
\hline C-multifocality & $28(47.5)$ & $26(44.1)$ & 0.853 \\
\hline C-tumor size $(\mathrm{cm})^{\dagger}$ & $2.6[2.3,3.0]$ & $2.7[2.5,3.5]$ & 0.068 \\
\hline Nodal metastasis & $27(45.8)$ & $29(49.2)$ & 0.854 \\
\hline Pathologic tumor size $(\mathrm{cm})^{+}$ & $2.3[2.1,3.0]$ & $1.5[0.6,2.2]$ & $<0.001$ \\
\hline Pathologic multifocality & $19(9.6)$ & $6(10.2)$ & 0.420 \\
\hline Poor differentiation histology & $3(5.1)$ & $1(1.7)$ & 0.538 \\
\hline $\mathrm{ER}(+)$ & $34(57.6)$ & $39(66.1)$ & 0.448 \\
\hline $\mathrm{PR}(+)$ & $31(52.5)$ & $32(54.2)$ & 1.000 \\
\hline HER2 (+) & $12(20.3)$ & $14(23.7)$ & 0.824 \\
\hline Ki-67 ( $\geq 50 \%)$ & $25(42.4)$ & $19(32.2)$ & 0.341 \\
\hline $\mathrm{DCIS}(+)$ & $40(67.8)$ & $26(44.1)$ & 0.016 \\
\hline $\mathrm{EIC}(+)$ & $15(25.4)$ & $5(8.5)$ & 0.027 \\
\hline Re-excision & $21(35.6)$ & $21(35.6)$ & 0.100 \\
\hline Success of BCS & $52(88.1)$ & $56(94.9)$ & 0.321 \\
\hline
\end{tabular}

$\mathrm{PS}=$ primary surgery; $\mathrm{PC}=$ primary chemotherapy; $\mathrm{C}$-microcalcification $=$ clinical microcalcification; C-multifocality= clinical multifocality (ultrasound); C-tumor size=clinical tumor size (ultrasound); $E R=$ estrogen receptor; $\mathrm{PR}=$ progesterone receptor; $\mathrm{HER} 2$ = human epidermal growth factor receptor 2-neu status; $\mathrm{DCIS}=$ ductal carcinoma in situ; $\mathrm{EIC}=$ extensive intraductal component; $\mathrm{BCS}=$ breast-conserving surgery.

*Mean \pm SD; ${ }^{\dagger}$ Performs a normality test, forces analysis as non-normal. 
regression model, the pathologic multifocality (odds ratio [OR], 4.56; $p=0.0142)$, high Ki-67 (OR, 0.7; $p=0.0243)$ and DCIS component (OR, 2.67; $p=0.0261)$ were the only variables that were independently associated with re-excision (Figure 1).

Table 3. In propensity score matching, re-excision versus non-re-excision

\begin{tabular}{|c|c|c|c|}
\hline & $\begin{array}{c}\text { Re-excision (-) } \\
(n=76) \\
\text { No. }(\%)\end{array}$ & $\begin{array}{c}\text { Re-excision (+) } \\
(n=42) \\
\text { No. }(\%)\end{array}$ & $p$-value \\
\hline Success of BCS & $76(100.0)$ & $32(76.2)$ & $<0.001$ \\
\hline Neoadjuvant chemotherapy & $38(50.0)$ & $21(50.0)$ & 1.000 \\
\hline Age $(y r)^{*}$ & $42.5 \pm 8.4$ & $43.5 \pm 7.3$ & 0.523 \\
\hline C-microcalcification & $22(28.9)$ & $15(35.7)$ & 0.581 \\
\hline C-multifocality & $31(40.8)$ & $23(54.8)$ & 0.206 \\
\hline C-tumor size $(\mathrm{cm})^{\dagger}$ & $2.6[2.3,3.0]$ & $2.9[2.4,3.5]$ & 0.095 \\
\hline Nodal metastasis & $32(42.1)$ & $24(57.1)$ & 0.170 \\
\hline Pathologic tumor size $(\mathrm{cm})^{\dagger}$ & $2.1[1.1,2.5]$ & $2.1[1.1,2.5]$ & 0.111 \\
\hline Pathologic multifocality & $5(6.6)$ & $11(26.2)$ & 0.007 \\
\hline Poor differentiation histology & $1(1.3)$ & $3(7.1)$ & 0.253 \\
\hline $\mathrm{ER}(+)$ & $42(55.3)$ & $31(73.8)$ & 0.074 \\
\hline $\mathrm{PR}(+)$ & $31(73.8)$ & $14(33.3)$ & 0.050 \\
\hline HER2 (+) & $16(21.1)$ & $10(23.8)$ & 0.909 \\
\hline Ki-67 ( $\geq 50 \%)$ & $35(46.1)$ & $9(21.4)$ & 0.014 \\
\hline $\operatorname{DCIS}(+)$ & $35(46.1)$ & $31(73.8)$ & 0.007 \\
\hline $\mathrm{EIC}(+)$ & $10(13.2)$ & $10(23.8)$ & 0.222 \\
\hline
\end{tabular}

Re-excision $(-)=$ do not re-excision; Re-excision $(+)=$ do re-excision; $\mathrm{BCS}=$ breast-conserving surgery; $\mathrm{C}-$ microcalcification $=$ clinical microcalcification; $\mathrm{C}$-multifocality $=$ clinical multifocality (ultrasound); $\mathrm{C}$-tumor size $=$ clinical tumor size (ultrasound); $\mathrm{ER}=$ estrogen receptor; $\mathrm{PR}=$ progesterone receptor; HER2 = human epidermal growth factor receptor 2-neu status; $\mathrm{DCIS}=$ ductal carcinoma in situ; $\mathrm{EIC}=$ extensive intraductal component. ${ }^{*}$ Mean $\pm \mathrm{SD}$; ${ }^{\dagger}$ Performs a normality test, forces analysis as non-normal.

\section{DISCUSSION}

Two large randomized trials: namely the National Surgical Adjuvant Breast and Bowel Project B-18 trial (NSABP) and the European Organization for Research and Treatment of Cancer 10902 trial (EORTC) confirmed the safety of preoperative chemotherapy for BCS. Preoperative chemotherapy produces similar results in terms of PFS, OS and local control compared with primary surgery in both clinical trials. In the NSABP B-18 trial, BCS before preoperative chemotherapy was more common (PS, 60\% vs. PC, 67\%; $p=0.002$ ) [14]. In EORTC 10902, tumor shrinkage due to preoperative chemotherapy led to an increase in BCS (PC, 22\% vs. PS, 21.3\%) [15]. Bonadonna et al. [16] reported that $76 \%$ showed good tumor shrinkage and $85 \%$ underwent BCS after primary chemotherapy. Christy et al. [17] reported that preoperative chemotherapy patients had the potential to have successful BCS (PC, 64\% vs. PS, 45\%). Among all patients undergoing initial BCS, a higher percentage in the primary chemotherapy group ended up with BCS as their final surgery (97\% vs. $81 \%, p<0.01$ ). Karanlik et al. [18] also demonstrated that of those who were scheduled for breast conservation surgery, patients who underwent primary chemotherapy was more likely to undergo BCS compared to those who did not (PC, 79/80 [99\%] vs. PS, 107/116 [92\%]). Our study included those who planned to receive BCS as primary operation or primary chemotherapy. The result showed that patients in the primary chemo-

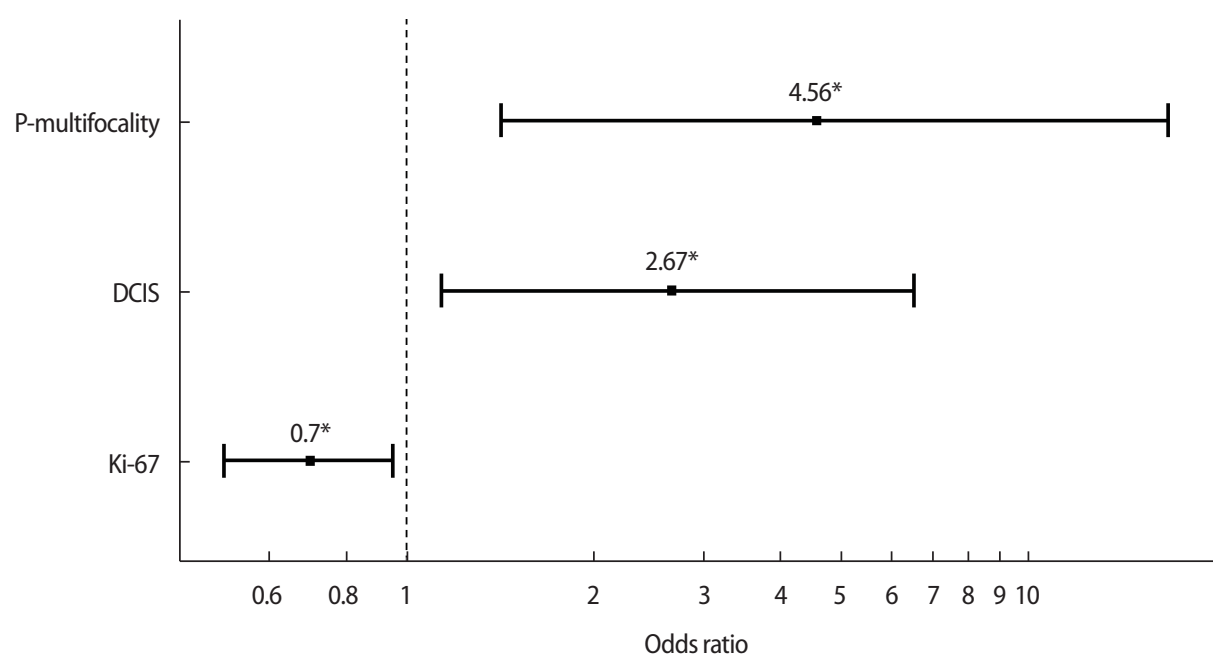

Figure 1. In propensity score matching, logistic regression model. In the final logistic regression model, the pathologic multifocality, high Ki-67, and ductal carcinoma in situ (DCIS) component were the only variables that were independently associated with re-excision. *Perform multiple logistic regression with variables which showed $p$-value less than 0.2 in univariate regression. Interpretation: larger than 0.05 , no overdispersion. 
therapy group have a higher success rate of BCS compared with that in the primary surgical group (PS, 178/197 [90.4\%]; PC, 56/59 [94.9\%]; $p=0.406)$. In most of the studies, the success rate of BCS was higher in those who underwent preoperative chemotherapy compared with those did not. However the reported success rate in those studies varied because of different affecting factor. More random attempts should be made to identify the variables.

The main goal of the BCS is to achieve tumor-free margins because tumor-involved margins have a high risk of local recurrence. A positive margin is among the most important predictors of increased risk for local recurrence. Therefore re-excision is required to obtain a negative resection margin.

Some papers report a lower rate of re-excision in primary chemotherapy. Christy et al. [17] showed that preoperative chemotherapy has a significantly higher incidence of negative margins (90\% vs. 55\%, $p<0.01)$ and a lower re-excision rate (6\% vs. $37 \%, p<0.01)$ compared with primary surgery. Karanlik et al. [18] also showed that primary chemotherapy was more likely to result in negative margins and less likely require re-excision ( $5 \%$ vs. $16 \%, p=0.02$ ). Others report a higher rate of re-excision in primary chemotherapy. Volders et al. [10] reported that primary chemotherapy patients received additional resection compared with primary surgery patients $(9.1 \%$ vs. $5.3 \%, p<0.001)$. Soucy et al. [19] demonstrated that $21.1 \%$ of primary chemotherapy patients had positive margins versus $17.9 \%$ in those who did not undergo primary chemotherapy $(p=0.52)$. Our study also reported a high re-excision rate in primary chemotherapy patient (PS, 67/197 [34.0\%]; PC, 21/59 [35.6\%]; $p=0.946)$.

A selection bias of surgeons in the decision of treatment methods existed between the two groups because this is a retrospective study. Before decision of treatment, in the age, clinical tumor size, and ultrasound multifocality of the two groups were significantly different. To reduce the effect of selection bias, propensity score matching-based analysis was performed. The result showed no difference between the two groups in terms of re-excision $(p=0.100)$ and the success rate of BCS was high in the primary chemotherapy group but not statistically significant (PS, 52/59 [88.1\%]; PC, 56/59 [94.9\%]; $p=0.321$ ). Preoperative chemotherapy did not affect the margin status and re-excision.

Degeneration patterns of tumors in response to chemotherapy can be variable, with residual tumor in mosaic pattern rather than circumferential. Therefore, the rate of re-excision is not lower in the first chemotherapy group than in the first surgery group. Recently Yang et al. [20] reported a regression pattern of tumor. This study presented that the clinicopathologic shrinkage modes were divided into two categories: concentric shrinkage mode (the longest diameter of the pathological residual tumors was less than $50 \%$ and $\leq 2 \mathrm{~cm}$ in comparison with the primary tumor before NAC) and non-concentric shrinkage mode. This study showed that patients with low primary tumor stage, PR (-) or mammographic malignant calcification (-) and with down-staging lymph node after NAC were more likely to present with concentric shrinkage mode after NAC ( $p<0.05$ for all). Consideration of the predictive factors affecting this shrinkage pattern in patient selection may increase the BCS success rate.

Risk factors associated with re-excision were investigated in several studies. Age, tumor size, tumor subtype, nodal status, EIC, and DCIS increase the rate of re-excision. In propensity score matching analysis, our study showed that the pathologic size $(p<0.001)$, DCIS $(p=0.016)$, and EIC ( $p=0.027)$ were significantly different between the two groups. PC group had smaller pathologic tumor size and less DCIS and EIC. However significant risk factors for re-excision are observed differently in each reported study [21-23]. Generalizing results is difficult due to the difference in the criteria of positive margin for performing re-excision. As such additional research is needed.

In this study, primary chemotherapy significantly reduced pathologic tumor size and increased BCS success rate, but did not reduce re-excision rate. Re-excision was more affected by clinicopathologic findings than chemotherapy. The study limitation is that the selected group is too small. Further analysis is needed to address this issue. In addition, understanding the pattern of regression of tumors and studying the accurate response and localization are necessary.

\section{CONFLICT OF INTEREST}

The authors declare that they have no competing interests.

\section{REFERENCES}

1. van Maaren MC, de Munck L, de Bock GH, Jobsen JJ, van Dalen T, Linn SC, et al. 10 year survival after breast-conserving surgery plus radiotherapy compared with mastectomy in early breast cancer in the Netherlands: a population-based study. Lancet Oncol 2016;17: 
1158-70.

2. Ye JC, Yan W, Christos PJ, Nori D, Ravi A. Equivalent survival with mastectomy or breast-conserving surgery plus radiation in young women aged $<40$ years with early-stage breast cancer: a national registry-based stage-by-stage comparison. Clin Breast Cancer 2015;15:390-7.

3. Agarwal S, Pappas L, Neumayer L, Kokeny K, Agarwal J. Effect of breast conservation therapy vs mastectomy on disease-specific survival for early-stage breast cancer. JAMA Surg 2014;149:267-74.

4. Veronesi U, Cascinelli N, Mariani L, Greco M, Saccozzi R, Luini A, et al. Twenty-year follow-up of a randomized study comparing breast-conserving surgery with radical mastectomy for early breast cancer. N Engl J Med 2002;347:1227-32.

5. Russo AL, Arvold ND, Niemierko A, Wong N, Wong JS, Bellon JR, et al. Margin status and the risk of local recurrence in patients with early-stage breast cancer treated with breast-conserving therapy. Breast Cancer Res Treat 2013;140:353-61.

6. Groot G, Rees H, Pahwa P, Kanagaratnam S, Kinloch M. Predicting local recurrence following breast-conserving therapy for early stage breast cancer: the significance of a narrow $(\leq 2 \mathrm{~mm})$ surgical resection margin. J Surg Oncol 2011;103:212-6.

7. Jobsen JJ, Riemersma S, van der Palen J, Ong F, Jonkman A, Struikmans $\mathrm{H}$. The impact of margin status in breast-conserving therapy for lobular carcinoma is age related. Eur J Surg Oncol 2010;36:17681 .

8. Gogescu G, Marinescu S, Brătucu E. Conserving surgery: balance between good cosmetic aspect and local disease control in incipient breast cancer. Chirurgia (Bucur) 2014;109:461-70.

9. Bulam H, Tuncer S, Ayhan S. Cosmetic outcome and percentage of breast volume excision in oncoplastic breast-conserving surgery: a proposal. World J Surg 2010;34:2260.

10. Volders JH, Haloua MH, Krekel NM, Negenborn VL, Barbé E, Sietses C, et al. Neoadjuvant chemotherapy in breast-conserving surgery: consequences on margin status and excision volumes: a nationwide pathology study. Eur J Surg Oncol 2016;42:986-93.

11. Vergine M, Scipioni P, Garritano S, Colangelo M, Di Paolo A, Livadoti $\mathrm{G}$, et al. Breast-conserving surgery after neoadjuvant chemotherapy in patients with locally advanced cancer. Preliminary results. G Chir 2013;34:254-6.

12. Cho JH, Park JM, Park HS, Park S, Kim SI, Park BW. Oncologic safety of breast-conserving surgery compared to mastectomy in patients receiving neoadjuvant chemotherapy for locally advanced breast cancer. J Surg Oncol 2013;108:531-6.

13. Peintinger F, Symmans WF, Gonzalez-Angulo AM, Boughey JC, Buzdar AU, Yu TK, et al. The safety of breast-conserving surgery in patients who achieve a complete pathologic response after neoadjuvant chemotherapy. Cancer 2006;107:1248-54.

14. Fisher B, Brown A, Mamounas E, Wieand S, Robidoux A, Margolese RG, et al. Effect of preoperative chemotherapy on local-regional disease in women with operable breast cancer: findings from $\mathrm{Na}$ tional Surgical Adjuvant Breast and Bowel Project B-18. J Clin Oncol 1997;15:2483-93.

15. van der Hage JA, van de Velde CJ, Julien JP, Tubiana-Hulin M, Vandervelden C, Duchateau L. Preoperative chemotherapy in primary operable breast cancer: results from the European Organization for Research and Treatment of Cancer trial 10902. J Clin Oncol 2001;19:4224-37.

16. Bonadonna G, Valagussa P, Brambilla C, Ferrari L, Moliterni A, Terenziani M, et al. Primary chemotherapy in operable breast cancer: eight-year experience at the Milan Cancer Institute. J Clin Oncol 1998;16:93-100.

17. Christy CJ, Thorsteinsson D, Grube BJ, Black D, Abu-Khalaf M, Chung GG, et al. Preoperative chemotherapy decreases the need for re-excision of breast cancers between 2 and $4 \mathrm{~cm}$ diameter. Ann Surg Oncol 2009;16:697-702.

18. Karanlik H, Ozgur I, Cabioglu N, Sen F, Erturk K, Kilic B, et al. Preoperative chemotherapy for $\mathrm{T} 2$ breast cancer is associated with improved surgical outcome. Eur J Surg Oncol 2015;41:1226-33.

19. Soucy G, Bélanger J, Leblanc G, Sideris L, Drolet P, Mitchell A, et al. Surgical margins in breast-conservation operations for invasive carcinoma: does neoadjuvant chemotherapy have an impact? J Am Coll Surg 2008;206:1116-21.

20. Yang T, Zhang ZP, Sun XY, Liu G, Mu DB, Wang YS. Shrinkage mode of the primary breast tumor after neoadjuvant chemotherapy analyzed with part-mount sub-serial sectioning and three-dimensional reconstruction technique. Zhonghua Zhong Liu Za Zhi 2016;38:270-6.

21. Gatek J, Vrana D, Melichar B, Vazan P, Kotocova K, Kotoc J, et al. Significance of the resection margin and risk factors for close or positive resection margin in patients undergoing breast-conserving 
surgery. J BUON 2012;17:452-6.

22. Tartter PI, Bleiweiss IJ, Levchenko S. Factors associated with clear biopsy margins and clear reexcision margins in breast cancer specimens from candidates for breast conservation. J Am Coll Surg 1997;185:268-73.
23. Smitt MC, Horst K. Association of clinical and pathologic variables with lumpectomy surgical margin status after preoperative diagnosis or excisional biopsy of invasive breast cancer. Ann Surg Oncol 2007;14:1040-4. 